ANDRÉS VALENCIA-ESCOBAR

UNIVERSIDAD PONTIFICIA BOLIVARIANA

MEDELLÍN, COLOMBIA

ANDRES.VALENCIA@UPB.EDU.CO

ALEJANDRO ZULETA

UNIVERSIDAD PONTIFICIA BOLIVARIANA MEDELLÍN, COLOMBIA

ALEJANDRO.ZULETA@UPB.EDU.CO

YULIANA AREIZA

SERVICIO NACIONAL DE APRENDIZAJE SENA

MEDELLÍN, COLOMBIA

YAAREIZA3@MISENA.EDU.CO

ESTEBAN CORREA

SERVICIO NACIONAL DE APRENDIZAJE SENA MEDELLÍN, COLOMBIA

CORREA54@MISENA.EDU.CO

\section{Diseño de bicicletas plegables en madera: una posibilidad real para el uso sostenible de un material renovable}

\author{
Wooden folding bicycle design: A real possibility for the \\ sustainable use of a renewable material
}

Resumen. La madera, entendido como un material sostenible, se usa desde finales del siglo XVIII para fabricar marcos de bicicletas y durante los últimos diez años su uso se ha incrementado. Sin embargo, no existen aún referencias científicas o comerciales de proyectos de bicicletas plegables de madera. Con base en esto, se propone un proyecto que busca diseñar una bicicleta plegable de madera que se pueda integrar a una estrategia de movilidad sostenible. Metodológicamente, se siguieron cuatro fases: Información, Formalización, Conformación y Validación. Se hizo un análisis de las características de modelos de bicicletas plegables existentes, se definieron los requerimientos de diseño, el patrón geométrico y el patrón estructural para el marco; se seleccionaron la madera y los componentes estándar y se diseñó el mecanismo de plegado. Se utilizaron prototipos a diferentes escalas para pruebas de ajuste dimensional con componentes y pruebas básicas de manejo para determinar maniobrabilidad y factores de ajuste ergonómico. Por último, se validó el comportamiento mecánico con un análisis por elementos finitos. Como resultado se obtuvo el diseño de un marco plegable en madera de teca compuesto por tres sistemas estructurales integrados, reforzados con insertos metálicos internos, basado en un triángulo trasero, una elipse central y un voladizo frontal.

Palabras clave: bicicletas de madera, bicicletas plegables, diseño de bicicletas, diseño con madera, materiales sostenibles.

Profesional del Sistema de Investigación, Desarrollo Tecnológico e Innovación - SENNOVA del SENA bajo el código SIGP:19218 y el Centro para la Investigación para el Desarrollo de la Innovación -CIDI- de la Universidad Pontificia Bolivariana bajo el radicado 259 C-08/18-35

Fecha de recepción: 27/07/2018

Fecha de aceptación: 03/10/2018

Cómo citar: Valencia-Escobar, A., Zuleta,

A., Areiza, Y. y Correa, E. (2018) Diseño

de bicicletas plegables en madera: una posibilidad real para el uso sostenible de un material renovable. RChD: creación y pensamiento, 3(5), 1-11.

DOI: $10.5354 / 0719-837 \times .2018 .50716$

Revista Chilena de Diseño,

RChD: creación y pensamiento

Universidad de Chile

2018, 3(5)

http://rchd.uchile.cl
Abstract. Wood, understood as a sustainable material, has been used since the end of the 18th century to manufacture bicycle frames and during the last ten years its use has increased. However, there are still no scientific or commercial references for folding wooden bicycle projects. Based on this, a project is proposed that seeks to design a folding wooden bicycle that can be integrated into a sustainable mobility strategy. Methodologically, four phases were followed: Information, Formalization, Conformation and Validation. An analysis of the characteristics of existing folding bicycle models was made, the design requirements, the geometric pattern and the structural pattern for the frame were defined. Wood and standard components were selected and the folding mechanism was designed. Prototypes were used at different scales for dimensional fit tests with components, basic driving tests to determine maneuverability and ergonomic adjustment factors. Finally, the mechanical behavior was validated with a finite element analysis and with experimental structural testing protocol. As a result, the design of a folding frame in teak wood composed of three integrated structural systems, reinforced with internal metallic inserts, based on a rear triangle, a central ellipse and a front overhang was achieved.

Keywords: bicycle design, design with wood, folding bicycles, sustainable materials, wooden bicycles. 


\section{Introducción}

Se sabe que actualmente el crecimiento y la densificación de la población en las diferentes ciudades generan, de forma proporcional, un aumento en el número de automóviles. De hecho, según información referenciada en la página web del Foro Económico Mundial y publicada por el grupo de investigación Bernstein, para 2040 se espera que el número de automóviles en las ciudades se haya duplicado (Nitch, 2016). Esta situación se convierte en una fuente de contaminación, congestión, accidentalidad, escasez de recursos y dependencia de fuentes energéticas no renovables. No obstante, se sabe que la bicicleta se ha convertido en una alternativa sostenible y sustentable para atender la demanda de medios de transporte en las ciudades y que esto ha impactado positivamente de manera directa los problemas mencionados (Nieuwenhuijsen \& Khreis, 2016; Rode, 2017; Vuchic, 2017).

En Colombia, cuyas ciudades capitales se presentan cada vez más congestionadas por el tráfico automovilístico, se han propuesto, cada vez con más fuerza y en más lugares, estrategias y políticas públicas que procuren por sistemas urbanos de movilidad sostenibles basados en la bicicleta como alternativa de medio de transporte autónomo para trayectos cortos, el que pueda ser integrado con los sistemas de transporte público existentes para trayectos largos (Reyes, Riaño, \& Castro, 2015; Pineda, 2016; Velásquez \& Hidalgo, 2017). Algunos ejemplos de lo mencionado, que se han presentado específicamente en Medellín, son el Sistema de Bicicletas Públicas del Valle de Aburrá ENCICLA y la optimización de la red vial de la ciudad en su búsqueda de una movilidad sustentable (Ospina, 2016).

Dentro de los modelos de bicicletas existentes, las plegables se presentan como las más apropiadas para articularse con estrategias multimodales de movilidad urbana (Krizek \& Stonebraker, 2010; Harris, 2011). La capacidad de disminuir su tamaño y un peso proporcionado a la capacidad de carga de una persona, hacen de este tipo de bicicletas un referente claro para la optimización de los desplazamientos urbanos integrados en ciudades altamente congestionadas. Existen muchos modelos comerciales de este tipo de bicicletas, y en ellas unos referentes claros de diseño son la relación peso/volumen en el estado plegado, las condiciones ergonómicas a la hora de moverse con la bicicleta a cuestas y la apariencia visual. Análisis científicos de una o varias de estas variables en proyectos de diseño de bicicletas plegables pueden verse, entre otros, en el trabajo de Tosi et al. (2012), Niu et al. (2014), Luo y Li (2014) y Tovey y Roy (2016). También pueden verse reflejadas en la información comercial de los nuevos desarrollos de las marcas existentes en el mercado, como Bromptom en Inglaterra, Tern en Taiwan y Dahon en Estados Unidos, las cuales son las más representativas. No obstante esta información, ninguno de ellos reporta el uso o usa la madera como material para el desarrollo del marco.

La madera ha sido utilizada desde finales del siglo XVIII como material para el diseño de marcos de bicicletas, inicialmente debido a su disponibilidad y al conocimiento que los artesanos tenían de él debido a la construcción de carruajes, y luego debido a sus condiciones estructurales y estéticas (Herlihy, 2004; Varela, 2006; Hadland y Lessing, 2014; Valencia-Escobar, 2015). Durante los últimos 10 años, y luego de un letargo de casi 100 años, la madera ha retomado un auge muy importante tanto en proyectos productivos comerciales, como es el caso de Renovo y Coonor Wood Bikes en Estados Unidos, Bouugh Bikes en Holanda o Axalco en España, entre muchos otros, como en 

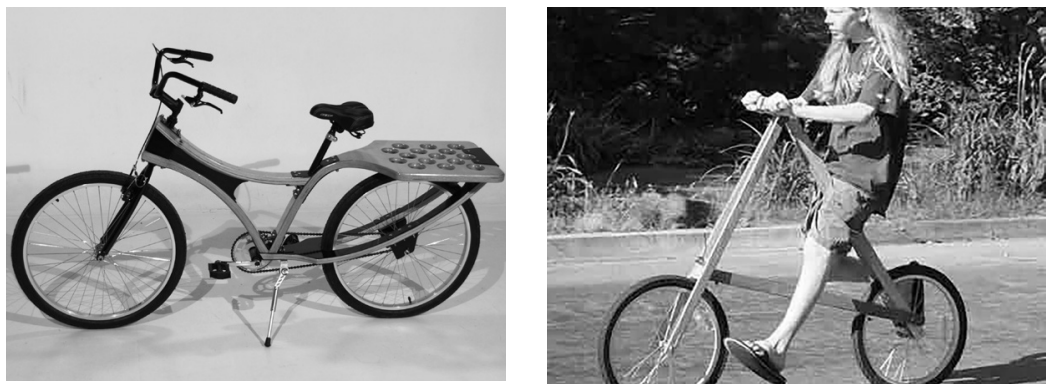

proyectos de diseño individuales que pueden verse en internet. Una prueba de este regreso y de su validez es la publicación del libro The wooden bicycle around the world, compilado por Kiriakos Iosifidis (2017), en el que se muestran cerca de 150 proyectos alrededor del mundo. Dentro de este renacer, la Universidad Pontificia Bolivariana, en cabeza de su Grupo de Investigación de Estudios en Diseño, se presenta como una de las pocas instituciones académicas a nivel mundial que demuestra un trabajo amplio y exitoso en torno al diseño de bicicletas con marcos de madera (Figura 1). Este trabajo se demuestra con la realización de diecisiete versiones de su curso de diseño de bicicletas de madera, en las cuales se han desarrollado más de cien modelos de bicicletas diferentes y que pueden ser vistos en internet en la dirección https://www.flickr.com/photos/valenciaandres/. Por su lado, el Grupo de Investigación Materiales para el Mobiliario del SENA, ha estado trabajando ampliamente en proyectos enfocados al uso tradicional e innovador de la madera como material para el diseño desde hace 8 años.

Es claro que en el mundo existen una gran cantidad de modelos de bicicletas de madera, no obstante, solo se han identificado dos aproximaciones a la tipología de bicicleta plegable. Una de ellas se basa en el mismo principio que tiene la bicicleta Strida y se presenta como un proyecto nombrado como "Hágalo usted mismo" con materiales y componentes en su mayoría adaptados (Figura 2a). El otro es un diseño desarrollado en Indonesia, denominado Garuda Baik, diseñada por Melisa Dawson y Troy Fitchu Tanalisan, que se ajusta al modelo de doble triángulo con un sistema de bisagras en la parte central del marco (Figura 2b). A pesar de lo anterior, ninguno de los dos proyectos presenta información relevante en cuanto a su proceso de diseño y análisis. Por otro lado, la madera cultivada responsablemente se considera hoy un material sostenible, que en el futuro, además, contribuirá al mejoramiento ambiental (Skog et al, 2015; UNECE, 2016). Colombia, por su ubicación geográfica y sus condiciones topográficas, se presenta como un país en el cual la industria maderera puede desarrollarse de manera activa y de hecho lo hace. En virtud de todo lo anterior, este proyecto, que tuvo como objetivo el diseño de un nuevo modelo de bicicleta plegable cuyo marco fuera de madera, se estructuró a través de una alianza estratégica entre los grupos de investigación mencionados. El interés de este trabajo es dar a conocer la metodología empleada para el diseño de una bicicleta plegable urbana con un marco de madera maciza, en el que se hace énfasis a los criterios de selección del material y la definición del patrón estructural del marco. Para esto, se tiene en cuenta la exploración del estado del arte y las aproximaciones en construcción y diseño de bicicletas en el área local de la ciudad de Medellín en Colombia.

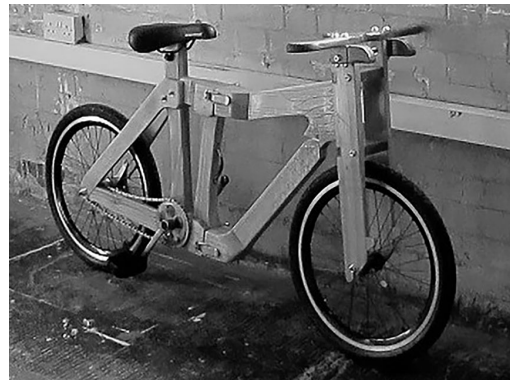

Figura 1. Uno de los más de 100 modelos de bicicletas con marco de madera laminada de pino que se han desarrollado en la UPB.

Figura 2a. modelo de bicicleta plegable en madera de estructura mono-triangular (Kabat, 2008). Figura 2b. modelo de bicicleta en madera con estructura de doble triángulo (Dawson y Fitchu, 2014). 
Figura 3. Modelos de bicicletas plegables analizados en la fase de información.

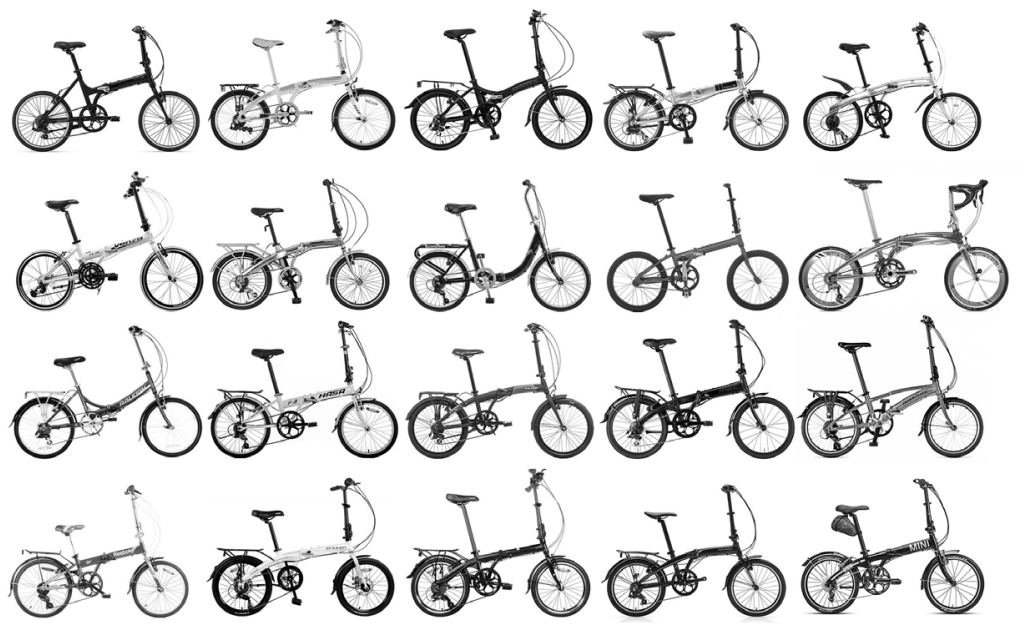

\section{Metodología}

El proyecto incluyó inicialmente una fase de información. En esta etapa se ejecutó un proceso documental de investigación para buscar definir los antecedentes y el estado del arte con respecto a dos aspectos fundamentales: los requerimientos de diseño para este tipo de bicicletas y los protocolos de validación funcional existentes. Lo anterior implicó una búsqueda en internet y en bases de datos científicas. En primera instancia, se hace la exploración detallada de veinte modelos de bicicletas plegables comerciales (Figura 3) y se obtienen los datos de componentes, mecanismos de plegado y medidas comunes de las bicicletas plegables y que presentan mayor interés para esta investigación. A partir de lo anterior, se procede con la definición del patrón geométrico, entendido como la disposición espacial bidimensional de ocho puntos de referencia: el eje trasero, el eje delantero, el eje centro, el inicio de la caña del asiento, el asiento, el inicio del tubo frontal, el final del tubo frontal y el manubrio, que posteriormente permitirá el desarrollo del patrón estructural. Además, se definió la ubicación del eje de rotación para el punto de plegabilidad. También se hizo un análisis del contexto de uso de la bicicleta y se desarrolló un proceso de conceptualización del proyecto buscando los referentes tanto para la marca como para la generación de la forma. Paralelamente, se trabajó en la identificación de los modelos de bicicletas rígidas con marco de madera más representativos en el mercado internacional y nacional y así buscar la denominación del tipo de madera o maderas que se usan para su fabricación. Esta información se organizó con el fin de construir el perfil de material que se requería para el proyecto en términos de las propiedades mecánicas, químicas y físicas de la madera. Luego, se hizo un proceso de selección de materiales a partir del modelo por análisis y similitud (Ashby y Johnson, 2013) para definir cuál era el tipo de madera más apropiada para el proyecto. La selección de la madera se realizó junto a un proceso de pruebas estructurales realizadas a la madera seleccionada y a una madera laminada de pino como referencia. Estas pruebas incluyeron ensayos de flexión a tres puntos tanto sobre los tableros comerciales como sobre piezas pegadas con los dos adhesivos que se esperaban utilizar en el ensamble final del marco. 


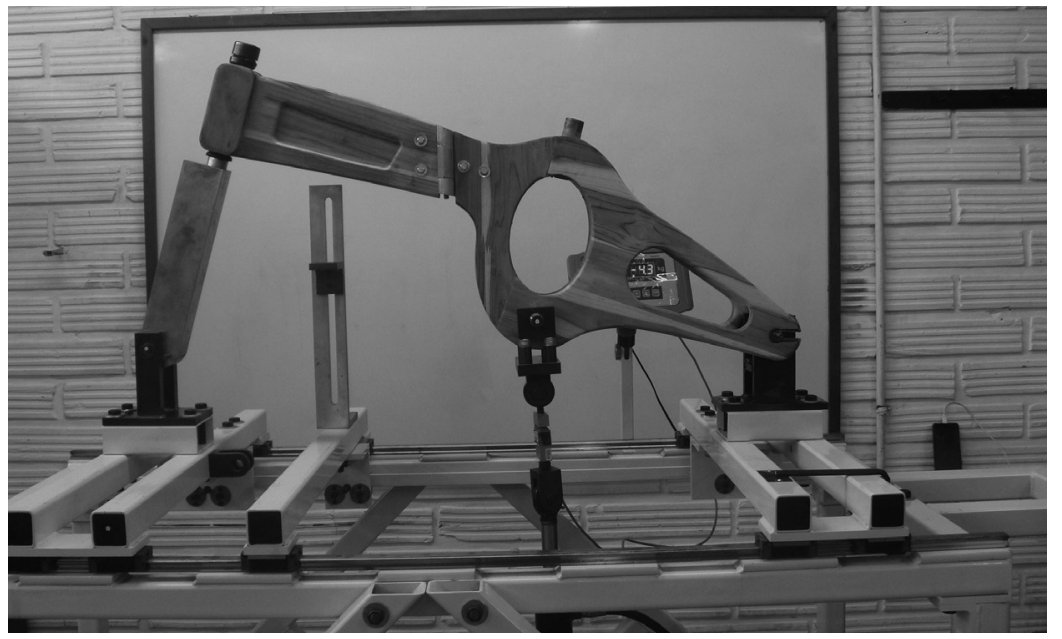

Seguidamente, se trabajó en la fase de formalización, en la cual se tomó la lista de requerimientos y el patrón geométrico de la fase anterior y se hizo el desarrollo bidimensional del patrón estructural en el que se tenían en cuenta aspectos técnicos y la información conceptual definida. Este patrón se establece a partir de la manera cómo se espera que se unan los puntos del patrón geométrico. Esta unión implica la definición de al menos tres variables: la longitud, la sección transversal y la sección longitudinal de cada uno de los elementos constitutivos del marco. Para ello, se trabajó con un proceso de ideación basado en el design thinking, con el fin de potenciar la generación de una gran cantidad de ideas. Luego, se filtraron las propuestas y se llegó a dos modelos finales que a su vez fueron evaluados con mayor profundidad luego de haber sido dibujados en dos dimensiones con ayuda de un software de CAD. De este proceso, se tomó la decisión de seleccionar un patrón y sobre él hacer variaciones.

Cada una de las variaciones fue modelada digitalmente y luego prototipada a escala en láminas de MDF. Con estos modelos, el patrón estructural final fue seleccionado y prototipado de nuevo en escala 1:1 en MDF. Este prototipo tuvo como objetivo definir condiciones geométricas y constructivas y validar la apariencia general de la bicicleta. Luego de detallar estas condiciones, se hizo un nuevo prototipo en madera laminada de pino para buscar una primera aproximación a la validación estructural. Estos dos primeros prototipos no contaron con el mecanismo de plegado, ya que se buscaba primero la identificación de los puntos críticos dentro de la estructura rígida. Después de las pruebas iniciales se prototipó un nuevo modelo en la madera seleccionada en la fase de información.

Si bien existen normativas internacionales para la validación estructural de marcos de bicicletas como las ASTM o las ISO, estas hacen referencia a configuraciones tradicionales desde la forma que no se ajustan al modelo desarrollado y por esta razón se optó por usar un protocolo de validación que fue diseñado para marcos de bicicletas de BMX (Valencia-Escobar, Zuleta y Rodríguez, 2017) y que, debido al tamaño del marco, fue posible utilizar. Este protocolo incluye una serie de pruebas que someten al marco a una condición de cargas similares a las que va a tener en servicio. El protocolo
Figura 4. Prototipo siendo sometido a una de las valoraciones estructurales en el banco de pruebas. Fuente: elaboración propia. 
Figura 5. Ensamble manual de uno de los modelos

Figura 6. Análisis por elementos finitos.

Izquierda: elemento central del marco.

Derecha: elemento central y trasero.
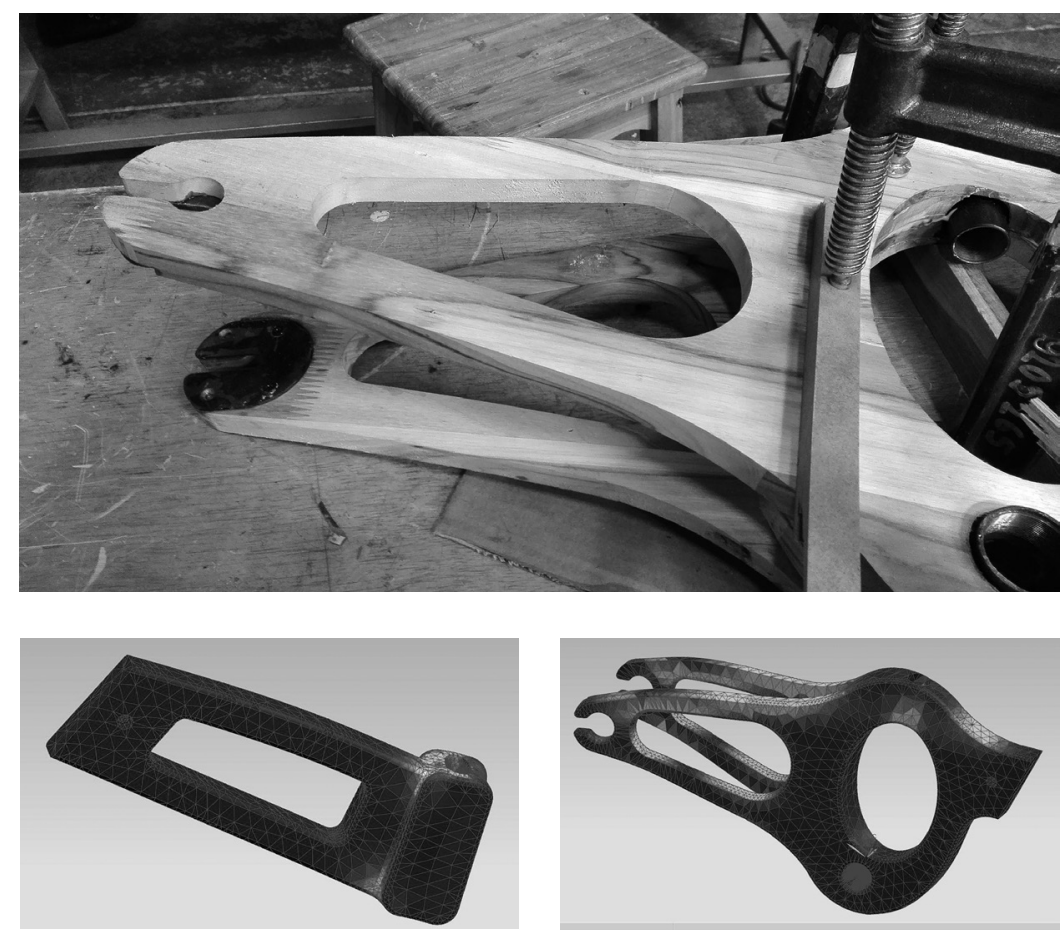

usa un banco de pruebas diseñado especialmente para la prueba (Figura 4). Con este último prototipo, inicia la fase conformación. Este nuevo prototipo fue sometido a un protocolo de pruebas estructurales, constructivas y operativas con el fin de afinar todas las características del mismo. En este punto ya se había desarrollado un mecanismo de plegado que fue incluido en el marco. Este proceso fue repetido cinco veces con prototipos mejorados hasta que se obtuvo un desempeño suficiente. Cada uno de los prototipos mencionados incluía cambios tanto formales como ajustes en la orientación de las vetas de la madera. La conformación se desarrolló inicialmente en una ruteadora de control numérico para la formación de piezas de madera y luego un proceso de ajuste dimensional y unión manual de piezas y componentes (Figura 5). Se utilizó un adhesivo a base de PVA para la unión entre piezas de madera y un adhesivo epóxico para la unión de las piezas metálicas con la madera.

Si bien las pruebas fueron constantes, se puede decir que la fase de validación se desarrolla en toda su profundidad con los prototipos finales que incluyen la madera seleccionada, las dimensiones establecidas y los componentes de refuerzo interno diseñados. Esta validación incluye, como se dijo, unos ensayos estructurales en un banco de pruebas, además de un proceso de análisis por elementos finitos que buscó la identificación de los puntos más críticos de la estructura (Figura 6). Además, se hicieron pruebas de ajuste ergonómico con seis usuarios, tres mujeres y tres hombres, cuya estatura se ajustaba a los rangos de percentiles que se esperaba abarcar con el diseño. Los hombres medían $1.84 \mathrm{~m}, 1.78 \mathrm{~m}$ y $1.69 \mathrm{~m}$ y las mujeres $1.62 \mathrm{~m}$, 1,60m y $1.58 \mathrm{~m}$. También se hicieron pruebas de manejo para determinar el nivel de funcionalidad. Finalmente, se hizo una validación perceptual con el fin de identificar el aporte que la forma y el material le dieron a la bicicleta. 


\section{Resultados y discusión}

Es posible definir un resultado específico de cada una de las fases metodológicas. De la fase de información se logró obtener el patrón geométrico requerido para el diseño del nuevo marco (Figura 7). Este patrón geométrico respondió a las condiciones funcionales requeridas para el ensamble de todos los componentes de la bicicleta y a las condiciones operativas relacionadas con la ergonomía de la bicicleta.

Se seleccionó también el tipo de material que se iba a utilizar. La teca (Tectona grandis) como tipo de madera y el tablón alistonado de $18 \mathrm{~mm}$ de espesor con unión por dedos, fue el resultado del proceso de selección de materiales por análisis. Se pudieron identificar al arce, al fresno, a la haya y al nogal como las especies más utilizadas en el mundo para fabricar marcos de bicicleta. En un análisis de la información técnica de estas maderas se estableció un valor promedio para las propiedades a tener en cuenta en la selección. Estos valores fueron: densidad de $0.66 \mathrm{~g} / \mathrm{cm}^{3}$, resistencia a la flexión MOR de 102.67 MPa y un módulo elástico MOE de $12.45 \mathrm{GPa}$. Los valores de estas mismas propiedades en la madera de teca son: densidad de $0.58 \mathrm{~g} /$ $\mathrm{cm}^{3}$, resistencia a la flexión MOR de 94.43 MPa y un módulo elástico MOE de 10.32 GPa. La comparación de estos valores muestra que la teca tiene una densidad $13 \%$ menor, una resistencia $8 \%$ menor y una rigidez $17 \%$ menor. Además, la teca tiene una resistencia natural a la intemperie y a los microorganismos mucho mejor que la mayoría de las maderas (Lukmandaru, 2017; Lukmandaru, 2017b), lo que la hace una excelente candidata para la fabricación de marcos de bicicleta. La teca no está incluida en las listas internacionales de especies en peligro y en Colombia se presenta como una de las maderas más productivas en términos de su relación entre los metros cúbicos obtenidos como madera tipo aserrío y madera tipo pulpa, y en la actualidad es la única especie que Colombia exporta (Alfonso, 2017).

Una vez seleccionada la teca como material, se desarrollaron pruebas estructurales de flexión a tres puntos con el fin de validar el desempeño mecánico de la lámina de $18 \mathrm{~mm}$ de espesor disponible comercialmente en Colombia en lo referente a la resistencia de las uniones longitudinales y transversales de los listones utilizados para su conformación y también para validar el comportamiento estructural de dos láminas unidas con adhesivos químicos comerciales. Esta prueba se desarrolló de manera comparativa para tener como referencia el comportamiento de una lámina completa de $18 \mathrm{~mm}$ y evaluar una unión pegada de dos láminas de $9 \mathrm{~mm}$. Se evaluaron la resina epóxica y el adhesivo de base PVA como mecanismos de unión. Se observó que para la lámina completa de teca la resistencia a la flexión -MORfue de 93 MPa mientras que el módulo de elasticidad -MOE- fue de 10.4 GPa. Para las dos láminas de teca adheridas con el adhesivo de base PVA el valor del MOR fue de 97.6 MPa y el MOE fue de 9.6 GPa y para las láminas adheridas con la resina epóxica el valor del MOR fue de 90.4 MPa y el MOE fue de 9.4 GPa. No fue evidente una diferencia estructural significativa entre los dos adhesivos, pero se encontró que la resina epóxica fragilizaba la unión y generaba una fractura catastrófica al momento de la falla, mientras que el PVA le permitía a la misma mantener las dos piezas adheridas aún en el momento en que se alcanzara la resistencia máxima del material.

En la fase de formalización se obtuvo como resultado un patrón estructural novedoso basado en una tijera trasera triangular unida a una zona central ovalada, la que a su vez se une a una zona frontal en voladizo (Figura 8). Este

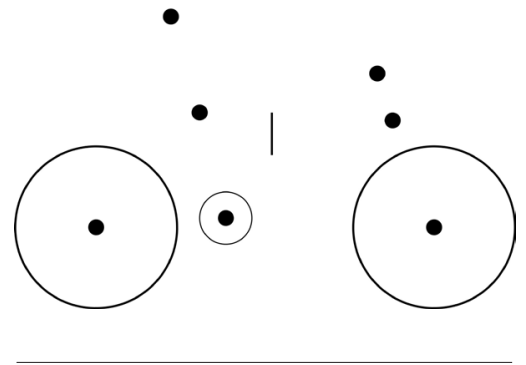

Figura 7. Patrón geométrico definido con base en los ocho puntos de referencia y el eje de rotación. Fuente: elaboración propia. 


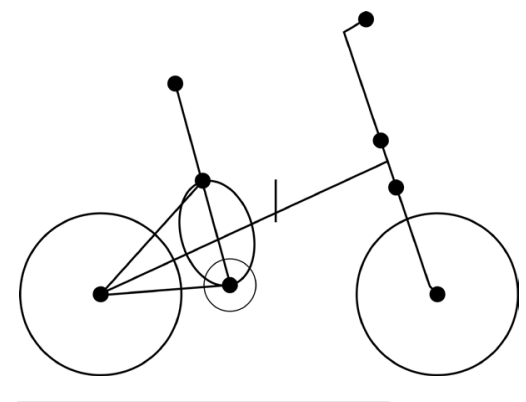

Figura 8. Patrón estructural definido Fuente: elaboración propia. patrón estructural responde tanto a los requerimientos estructurales como estéticos del proyecto y se ajusta a la restricción dada por la presentación laminar del material seleccionado. Una de las ventajas de este patrón se relaciona con el hecho de incluir una zona central ovalada que permite distribuir los esfuerzos de compresión y flexión generados en la zona del sillín y en la zona del eje central de manera más eficiente debido a esta configuración formal. Este tipo de patrones no tendrían viabilidad productiva con las materias primas tubulares tradicionales encontradas en los materiales metálicos como las aleaciones de acero y las aleaciones de aluminio.

También se obtuvo el diseño de una bisagra con un mecanismo de ajuste que se adaptó de manera precisa al diseño formal y estructural del marco (Figura 9). Esta bisagra se fabricó en aleación de aluminio 7075 T6.

En la fase de conformación se obtuvo un prototipo $100 \%$ funcional de una bicicleta plegable cuyo marco fue fabricado en madera de teca (Figura 10). El marco fue reforzado internamente con platinas de acero cold rolled 1020 en las zonas en las que había la necesidad de incluir piezas metálicas como en el frente de dirección, el eje centro y en la zona en la que el tubo del asiento entra en el marco. El prototipo final permite la variación en la altura del asiento con el fin de atender usuarios de diferentes estaturas. Luego de realizar un protocolo de validación estructural se alcanzó un nivel aceptable de desempeño estructural que permitió hacer pruebas de uso en condiciones reales de operación. La validación ergonómica (Figura 11) dio como resultado que la implementación de la fórmula del 1.09 (Burke, 2003) para el ajuste de la altura del asiento fue adecuada y en los usuarios evaluados la postura obtenida en la bicicleta fue la adecuada. Esta condición de diseño implica que la distancia medida entre el eje de los pedales y la parte superior del asiento debe ser el $90 \%$ de la altura de la entrepierna del usuario.

Las pruebas de percepción finales fueron realizadas con el prototipo mostrado en la Figura 12. Los usuarios involucrados en esta prueba manifestaron reacciones positivas al ver e interactuar con la bicicleta, indicaron percepciones sensoriales desde lo táctil y lo visual que justifican el uso del material. Por último, la validación técnica permitió por un lado ratificar un protocolo de pruebas estructurales que había sido inicialmente propuesto para marcos de bicicletas de $\mathrm{BMX}$, y por otro, combinar los análisis computacionales con las pruebas físicas en función de la optimización del diseño.

\section{Conclusiones}

La madera, vista como un material sostenible y asociado a una industria con un enorme crecimiento en países en vía de desarrollo como Colombia, es una materia prima de alta calidad que tiene un potencial enorme para ser aplicada en proyectos de diseño innovadores que rompan los paradigmas tanto de la forma como de la función. Las maderas tropicales, como la teca, se reconocieron como sustitutos adecuados para las maderas que crecen por fuera de los trópicos, inclusive aportan valores agregados como la resistencia natural a la intemperie y a las plagas. Sin embargo, se puede concluir que el trabajo con este material en el proyecto de diseño de un sistema dinámico como una bicicleta, implica tener en cuenta las propiedades del material desde una visión sistémica que solo puede ser validada con un proceso experimental sistemático y profundo.

La interacción multidisciplinar fue la clave para el éxito del proyecto. Una mirada plural atendida desde la Ingeniería y desde el Diseño Industrial le aporta 

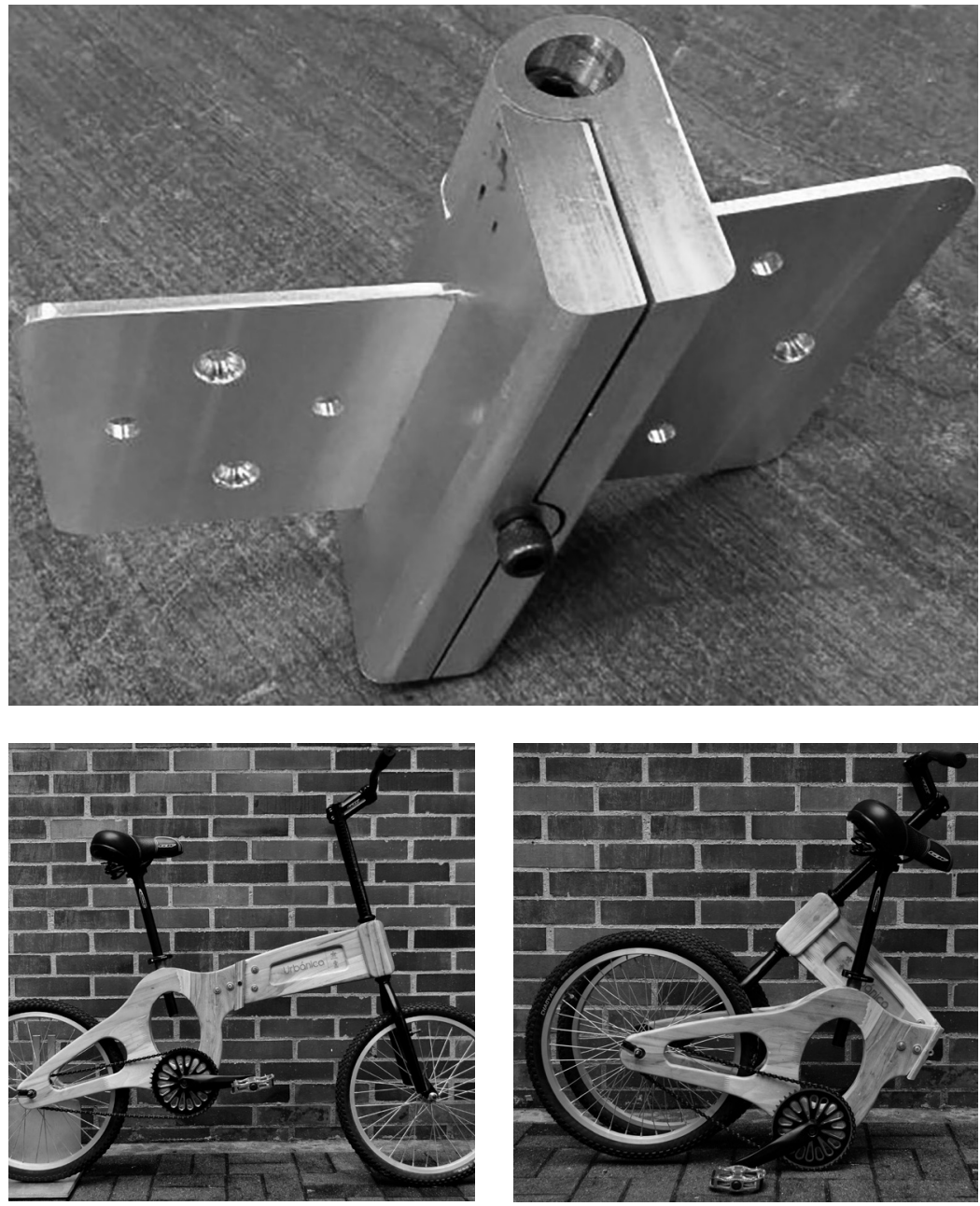

al proceso una fortaleza que le permite atender todos los requerimientos de un producto complejo como una bicicleta plegable.

Se pudo reconocer que la madera representa un factor de innovación categórico en cuanto a sus propiedades sensoriales se refiere, lo que invita a los usuarios a interesarse significativamente por el producto.

\section{Agradecimientos}

Los autores agradecen a la Universidad Pontificia Bolivariana y al SENA por su apoyo económico y logístico para el desarrollo del proyecto. Además, a William Tibavija, Sergio Soto, Laura Marín y Laura Osorno por su apoyo y trabajo incansable en las diferentes etapas del trabajo. Este proyecto fue financiado con recursos de la Dirección de Formación Profesional del Sistema de Investigación, Desarrollo Tecnológico e Innovación - SENNOVA del SENA bajo el código SIGP:19218 y con recursos del Centro para la Investigación para el Desarrollo de la Innovación -CIDI- de la Universidad Pontificia Bolivariana bajo el radicado $259 \mathrm{C}-08 / 18-35$.
Figura 9. Bisagra utilizada en el mecanismo de plegabilidad central. Fuente: elaboración propia. Figura 10. Prototipo final, estado desplegado. Fuente: elaboración propia.

Figura 11. Prototipo final, estado plegado. Fuente: elaboración propia. 
Figura 12. Análisis ergonómico postural y antropométrico con usuarios.

Fuente: elaboración propia.

Figura 13. Prototipo final acondicionado con todas las partes y accesorios.

Fuente: elaboración propia.
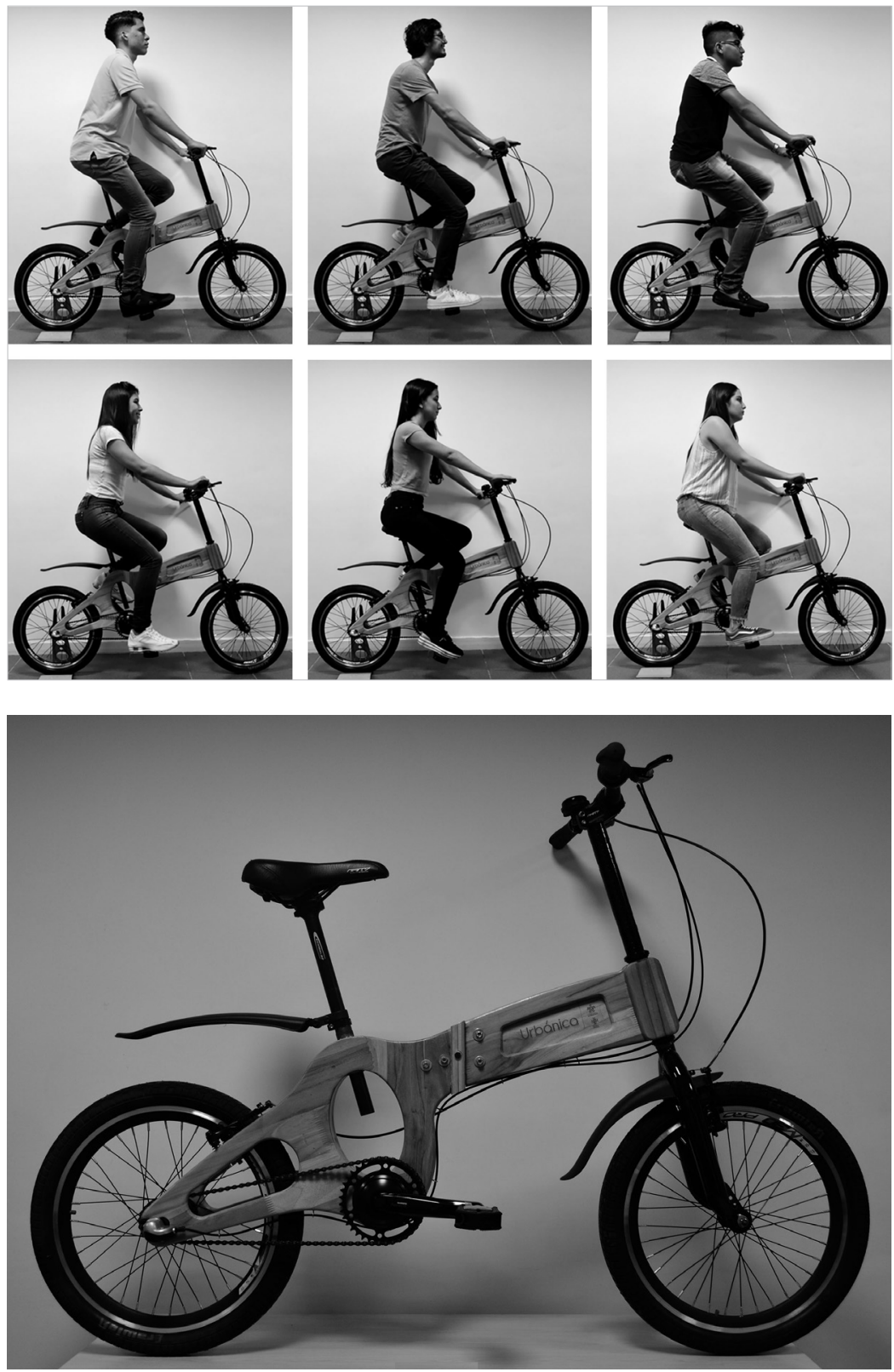


\section{Referencias bibliográficas}

Alfonso, K. (22 de abril de 2017). Más de $40 \%$ de la demanda de madera de la industria proviene de plantaciones ilegales. La República. Recuperado de www.larepublica.co.

Ashby, M. F., \& Johnson, K. (2013). Materials and design: the art and science of material selection in product design. Butterworth-Heinemann.

Burke, E. (2003). High-tech cycling. Human Kinetics.

Dawson, M y Fitchu, T. (2014). Teras. Recuperada de http://melisadawson.wixsite.com/sepedakayulipat en abril de 2018.

Hadland, T., \& Lessing, H. E. (2014). Bicycle design: An illustrated history. Mit Press.

Harris, K. W. (2011). Bike to the Future. The Futurist, 45(2), 25. Herlihy, D. V. (2004). Bicycle: the history. Yale University Press. Krizek, K., \& Stonebraker, E. (2010). Bicycling and transit: A marriage unrealized. Transportation Research Record: Journal of the Transportation Research Board, (2144), 161-167.

Kabat, Tom. Wooden Bikes. Recuperada de www.woodenbikes.com en abril de 2018.

Iosifidis, K. (2017). The wooden bicycle around the world. Images Publishing.Mulgrave.

Lukmandaru, G. (2017). Antifungal activities of certain components of teak wood extractives. Jurnal IImu dan Teknologi Kayu Tropis, 11(1), 11-18.

Lukmandaru, G. (2017). The natural termite resistance of teak wood grown in community forest. Jurnal IImu dan Teknologi Kayu Tropis 11(2), 131-139.

LUO, H. P., \& LU, H. Y. (2014). Design of Multifunctional Folding Bicycle. Mechanical Engineering \& Automation, 5, 030.

Nitch, M. (2016). The number of cars worldwide is set to double by 2040 . World Economic Forum. Recuperado de https://www.weforum.org/agenda/2016/04/thenumber-of-cars-worldwide-is-set-to-double-by-2040.

Niu, H. W., Yin, Y. C., Zhao, J. S., \& Chen, X. P. (2014). Innovative Design of the Portable Manually-Steered Folding Bicycle. In Advanced Materials Research (Vol. 889, pp. 212-216). Trans Tech Publications.

Ospina, G. (14 de julio de 2016). "Medellín busca una movilidad sustentable". El Colombiano. Recuperado de http://www.elcolombiano.com.

Pineda Jaramillo, J. (2016, June). Sustainability in urban transport plans. Case study: Monorail in a hillside area in Medellín. In XII Congreso de ingeniería del transporte. 7,8 y 9 de junio, Valencia (España) (pp. 1728-1734). Editorial Universitat Politècnica de València.
Nieuwenhuijsen, M. J., \& Khreis, H. (2016). Car free cities: pathway to healthy urban living. Environment international, 94, 251-262.

Reyes, L. V. R., Riaño, D. B., \& Castro, D. Q. (2015). BICISTEMA, Bicycle Transport System for Bogotá, Columbia. In European Transport Conference 2015Association for European Transport (AET).

Rode P. et al. (2017) Accessibility in Cities: Transport and Urban Form. In: Meyer G., Shaheen S. (eds) Disrupting Mobility. Lecture Notes in Mobility. Springer, Cham.

Roh, J., Hyeong, J., \& Kim, S. (2018). Influence of folding mechanism of bicycles on their usability. Applied ergonomics, 69, 58-65.

Skog, K. E., Wegner, T. H., Bilek, E. T., \& Michler, C. H. (2015). Desirable properties of wood for sustainable development in the twenty-first century. Annals of Forest Science, 72(6), 671-678.

Tosi, F., Belli, A., Rinaldi, A., \& Tucci, G. (2012). The Intermodal Bike: multi-modal integration of cycling mobility through product and process innovations in bicycle design. Work, 41 (Supplement 1), 1501-1506.

Tovey, M., \& Roy, R. (2016). Bicycle Design: Creativity and Innovation. In Design forTransport (pp. 189-222). Routledge.

UNECE. (2016). The 21st Century will be the century of Wood. Recuperado de https://www.unece.org/ info/media/presscurrent-press-h/forestry-and-timber/2016/the-21st-century-will-be-the-century-ofwood/doc.html

Valencia-Escobar, A. (2015). Si la historia hablara de la bicicleta. Revista Biker. (7), pp. 42-46.

Valencia-Escobar, A., Zuleta, A. y Rodríguez, C. (2017). El análisis estructural jerárquico como herramienta para el rediseño de un marco de bicicleta BMX. En VIII Congreso Internacional de Ingenierías Mecánica y Mecatrónica y VI de Materiales, Energía y Medio Ambiente (CIMM-2017). Medellín, Colombia.

Varela, I. (2006). The History and Technological Significance of the Bicycle. Economics, 354.

Velásquez J.M., Hidalgo D. (2017) Colombia. In: Pojani D., Stead D. (eds) The Urban Transport Crisis in Emerging Economies. The Urban Book Series. Springer, Cham. Vuchic, V. (2017). Transportation for livable cities. Routledge. 